\title{
Data Analytics for Smart Decision-Making and Resilient Systems
}

\author{
Benjamin Blau, Clemens van Dinther, Christoph M. Flath, Rico Knapper, \\ and Daniel Rolli
}

\begin{abstract}
In a networked world, companies depend on fast and smart decisions, especially when it comes to reacting to external change. With the wealth of data available today, smart decisions can increasingly be based on data analysis and be supported by IT systems that leverage AI. A global pandemic brings external change to an unprecedented level of unpredictability and severity of impact. Resilience therefore becomes an essential factor in most decisions when aiming at making and keeping them smart. In this chapter, we study the characteristics of resilient systems and test them with four use cases in a wide-ranging set of application areas. In all use cases, we highlight how AI can be used for data analysis to make smart decisions and contribute to the resilience of systems.
\end{abstract}

\section{Introduction}

Our global economy today is a highly interconnected system. According to Ricardo (1817), open markets lead to comparative cost advantages. Companies specialize and exploit local advantages (e.g., skilled workers or cost advantages due to cheap

\author{
B. Blau \\ SAP SE, Walldorf, Germany \\ e-mail: benjam.blau@sap.com \\ C. van Dinther $(\bowtie)$ \\ ESB Business School at Reutlingen University, Reutlingen, Germany \\ e-mail: clemens.van_dinther@reutlingen-university.de \\ C. M. Flath \\ Universität Würzburg, Würzburg, Germany \\ e-mail: christoph.flath@uni-wuerzburg.de \\ R. Knapper \\ anacision $\mathrm{GmbH}$, Karlsruhe, Germany \\ e-mail: rico.knapper@anacision.de \\ D. Rolli \\ Conemis AG, Karlsruhe, Germany \\ e-mail: daniel.rolli@conemis.com \\ (C) The Author(s) 2021 \\ H. Gimpel et al. (eds.), Market Engineering, \\ https://doi.org/10.1007/978-3-030-66661-3_13
}


raw materials or low wages). At the same time, companies are under high price and innovation pressure in global competition, which is difficult to counter without a strong partner network. For this reason and due to specialization, there is a dependency of the companies among each other in a value-added network. Close cooperation between companies is also possible due to the global communication infrastructure, as information can be passed on and shared quickly. At the same time, this presents companies with the challenge of being able to process information quickly and effectively, since the dynamics of the markets also require companies to be able to make decisions quickly. First of all, this includes the ability to perceive a change in the market situation on the basis of existing or observable information or, in the best case, to foresee it early on. In addition to the ability to observe and process information, companies use information not only to recognize changes but also to prepare decisions or even to make automated decisions. This means that those companies have a competitive advantage, if they have mastered fast and effective data/information processing and are able to benefit from it, and if they have the organizational capability to react quickly, appropriately, and agilely to changes.

The world economic crisis caused by the COVID-19 pandemic in 2020 highlights both the global economic interdependencies and the importance of rapid response. Resilience is an adaptive capacity, i.e., the ability of a system to stabilize itself after external shocks (Holling 2001, p. 394). Resilient systems are adaptable, i.e., companies are able to react to external events. In order to make the right decisions in a crisis situation, a good information base is required. Modern methods of information processing can help to create this information base. Thus, self-learning algorithms can help to process and deal with the changed situation. However, adaptive systems usually require a certain amount of adaptation time, because on the other hand, too much sensitivity of the system would lead to permanent change-resistance to adaptation is certainly desirable to a certain degree. This shows the dilemma of adaptive systems. Based on this, the research question for the development of information systems is how and where data analytics can be used for smart decisions and thus make systems more resilient. Our chapter is therefore structured as follows. First, in Sect.2, we look at the state of the art with respect to data science and smart decision-making and discuss in Sect. 3 how data analysis can contribute to the design of resilient systems. Sections 4, 5, 6, and 7 deal with use cases in which data analytics contributes to improved information processing. Section 8 summarizes our results in a conclusion and provides an outlook.

\section{Data Analysis for Decision-Making}

Decisions are fundamental tasks of management and determine the success and failure of an organization (Edwards et al. 2000). Decisions are based on current, relevant, and accurate information that must be collected or newly created. Organizations have a large amount of structured and unstructured data that is still far too often unused. It should be noted that decisions are not always easy to make, 
especially in complex situations, where alternatives are difficult to compare, or where there is uncertainty about the available information (e.g., because it relates to the future). Therefore, it is important to prepare and support decisions with appropriate information and information systems.

Data analysis can help to extract new information from the available data. The technical development of the last decades enables a powerful data analysis. Due to the growing computing power and the advanced algorithms of artificial intelligence, it is possible to process and analyze large amounts of data and thus generate new information. In this context, we understand the term smart decisionmaking as the ability to prepare or automatically make decisions with the help of intelligent algorithms for data analysis. In the literature, there are numerous synonymous uses of the term smart decision-making, e.g., intelligent decisionmaking (Phillips-Wren 2012), algorithmic decision-making (Bader and Kaiser 2019), automated decision-making (Araujo et al. 2020), autonomous decisionmaking (Kusiak et al. 2000), augmented decision-making (Burton et al. 2020), AI-based decision-making (Shrestha et al. 2019), and computational intelligencebased decision-making (Lakhmi C. Jain 2009). Already in 2006, Phillips-Wren and Jain (2006) pointed out the revolution in decision-making with the help of AI, which is capable of coordinating the provision of data, analyzing data trends, making forecasts, or quantifying uncertainty. Thus, decision-making in complex situations can be improved (Akerkar 2019, p. 6).

However, Stubbs (2014, p. 5) points out that data analysis alone does not create added value but that a benefit only comes from the action, i.e., the decision and the resulting action. Companies typically show different stages of development with regard to the ability to process information. Analytics maturity models can be useful in determining the level of development and skills. IBM has already presented such a model in 2013. Maturity refers both to the ability to use data and to how companies can use this information to their own advantage. Figure 1 shows the IBM information and analytics maturity model with the five maturity levels.

In principle, we can distinguish between decision support and automation. At the level of "differentiating," the IBM model shows the use of predictions, i.e., the use of historical data to identify trends or for pattern recognition as well as the use of forecast models. This is also known as predictive analytics. At the "breakaway" level, it is referred to as the use of prescriptive analytics with realtime pattern-based strategies. Here, prescriptive analytics means "going beyond the forecast to actually determine the optimal decision to make" (Frank Stein and Arnold Greenland 2014, p. 35). For this purpose, for example, simulation models can be developed. Such simulation models can be based on intelligent agents (van Dinther 2007, 2008). AI technologies are used for both predictive and prescriptive analytics. For example, fuzzy logic, expert systems, case-based reasoning, evolutionary algorithms, intelligent agents, random forests, artificial neural networks, or other machine learning algorithms are used. Lepenioti et al. (2020) provide a good overview of different AI methods for analytics. We illustrate in several use cases how such techniques can assist smart and resilient decisionmaking. 


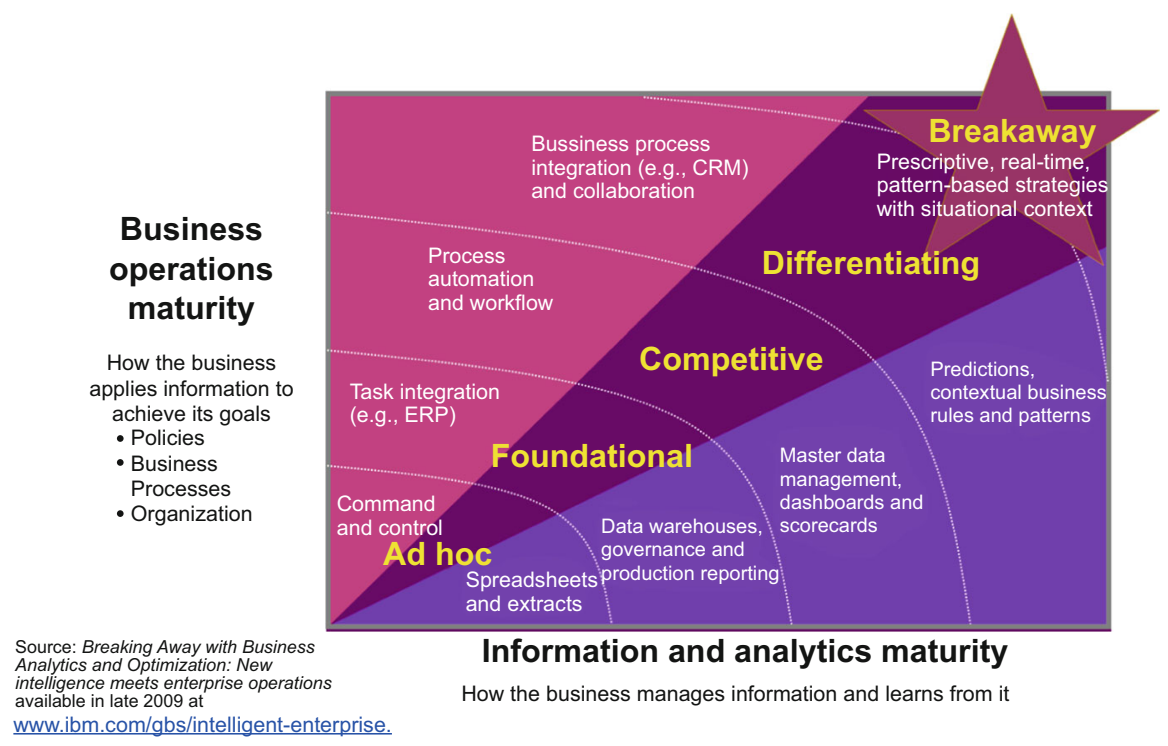

Fig. 1 IBM analytics maturity model

\section{Resilient Systems and the Supporting Role of Data Analytics}

Resilient systems are known to us from nature. Therefore, the terms "resilience" and "system" first appear in the context of ecosystems (Gunderson and Holling 2002). Folke et al. (2002, p.438) describe resilience in socioecological systems in relation to $(i)$ the magnitude of shock that the system can absorb and remain within a given state, (ii) the degree to which the system is capable of self-organization, and (iii) the degree to which the system can build capacity for learning and adaptation. The term resilience was later on picked up in systems engineering by Jackson and Ferris (2013). The definition mainly builds upon the three criteria as shown in Table 1 which differ in aspect 2 and 3 from the aspects provided by Folke et al. (2002).

While these criteria are derived from engineering systems, it is feasible and meaningful to abstract them to complex systems in general (e.g., social systems, large companies as described in Cheema-Fox et al. 2020, service value networks as described in Blau et al. 2009). With the recent example of the COVID-19 crisis,

Table 1 Criteria of a resilient system based on Jackson and Ferris (2013)

\begin{tabular}{l|l}
\hline Criteria & Description \\
\hline Flexibility & The ability of the system to adapt to a threat \\
\hline Tolerance & The ability of the system to degrade gracefully in the face of a threat \\
\hline Cohesion & The ability of the system to act as a unified whole in the face of a threat \\
\hline
\end{tabular}


Table 2 Strategy map along resilience criteria and target audiences

\begin{tabular}{l|l|l|l}
\hline & $\begin{array}{l}\text { Customer } \\
\text { experience }\end{array}$ & $\begin{array}{l}\text { Employee } \\
\text { experience }\end{array}$ & $\begin{array}{l}\text { Stakeholder } \\
\text { experience }\end{array}$ \\
\hline Flexibility & $\begin{array}{l}\text { Meet customers } \\
\text { where they are }\end{array}$ & $\begin{array}{l}\text { Prepare for } \\
\text { "unknown unknowns" }\end{array}$ & $\begin{array}{l}\text { Move quickly to } \\
\text { preserve trust }\end{array}$ \\
\hline Tolerance & $\begin{array}{l}\text { Listen, care, and } \\
\text { support }\end{array}$ & $\begin{array}{l}\text { "Show the beach, } \\
\text { not the trip" }\end{array}$ & $\begin{array}{l}\text { Build up predictable } \\
\text { revenue streams }\end{array}$ \\
\hline Cohesion & $\begin{array}{l}\text { Make customer success } \\
\text { the ultimate metric }\end{array}$ & $\begin{array}{l}\text { End-to-end processes } \\
\text { beyond functions }\end{array}$ & $\begin{array}{l}\text { Build sustainable } \\
\text { business }\end{array}$ \\
\hline
\end{tabular}

the relevance and importance of resiliency of complex systems to be able to deal with external shocks has been proven in an unplanned and unprecedented macroeconomic "experiment."

While the criteria of resilient systems have proven to be essential for companies to persist and perform in the face of external shocks, it is important to create and implement a strategy map outlining measures that turn the criteria into action tailored for each major stakeholder. Table 2 shows such a strategy map of SAP.

The pandemic caused by COVID-19 shows the vulnerability of global supply chains (Golan et al. 2020; Pierre Haren and David Simchi-Levi 2020; Sharma et al. 2020) and the need for resilient systems and analytics. Simulations of the supply chain help to better understand this vulnerability (Ivanov 2020) and to design resilient systems.

Focusing on customer experience, "meeting customers where they are" is essential to quickly adapt to changing needs in B2B (Hartmann and Lussier 2020). Accelerated roadmaps for digital touch points, digital supply chains, and a seamless digital front-to-back-office experiences are essential to cater for a changed buying behavior. This requires thorough data analysis of customer behavior along the full life cycle. This allows for early prediction of altering patterns and adaptation of the roadmap accordingly. Customer data analytics is applied to forecast sales (cp. Fan et al. 2017; Foxall 2017; Loureiro et al. 2018). Increased system tolerance and "acting as a whole" can be achieved by implementing team incentives for customer success across all roles of the value chain. The design of such incentive schemes requires modeling of historic data and simulation of worst-, mid-, and best-case scenarios. To drive change, especially in sales, requires a proactive winner-loser analysis. This helps to eliminate uncertainty related to fear of loss of control in the affected group.

Catering for employee experience in a resilient manner is another important pillar to assure safety and retain productivity. When it comes to flexibility, i.e., the ability of the system to adapt to the threat, agile employee-related processes are important to deal with the unknown unknowns. There is a large portion of events and external shocks that are beyond predictability/the horizon of possibly anticipated threats. Only agile processes can adapt quickly in case of non-anticipated unknown influences. Event-based and data-driven systems can help to detect unknown unknowns quickly and automatically adapt agile processes to the new 
circumstances. Tolerance according to the definition of Jackson and Ferris (2013) in the context of employee experience can be achieved by a clear target state that demonstrates the benefits for the employees beyond times of crisis. The design of such a target state requires data-intense analysis and simulations based on historic and external data sources. The "beach" is an analogy for such a target state. It is of utmost importance from a change management perspective to mainly communicate that "beach" instead of the process to get there, i.e., the related "trip." In addition to flexibility and tolerance, cohesion is an important aspect especially among the employee population to achieve resiliency. To foster collaboration and a team identity, end-to-end processes beyond functional silos are a key ingredient. For example, if companies manage to establish streamlined marketing to sales handover processes supported by event- and data-based routing systems and respective team incentives such as revenue contribution, cohesion will be a logical end result.

The third important experience customers need to cater for is the shareholder experience. Flexibility needs to be demonstrated by quickly adapting to the new normal, that is, radically rethinking existing structures and processes (e.g., changing service delivery model from on-site to almost purely remote within less than a month which can only be achieved by eliciting customer expectations based on vast data lakes). The ability to keep a stable and believable market guidance while quickly adapting to safeguard the top and bottom lines is crucial for resilient companies. Tolerance, i.e., the ability of the system to degrade gracefully in the face of a threat-in other words, endurance, can be achieved by building and sustaining predictable revenue streams radically shifting to subscription- or consumption-based commercial models. Demonstrating cohesion to shareholders requires fundamentally relevant and long-term objectives such as sustainability measures and ambitions. Profitable sustainability and sustainable profitability as a common goal rallies the troops moving as a single unit.

The following use cases illustrate the main aspects of resilient systems, flexibility, tolerance, and cohesion and how they can be achieved with data-driven decision-making based on real-world scenarios.

\section{Use Case 1: Improving Employee Experience Through the Listen-Learn-Act Approach and Reasoning on Semi-structured Data}

For many employees, their personal well-being and a good work-life balance are now more important than the basic employment contract conditions such as salary, company car, etc. Not least because of this development, there has been a shift from a job market to an applicant market for some time. Companies must therefore address these points and ensure satisfaction not only during the application phase but also during the course of their employees' work-at the same time, of course, increase work output as much as possible. The listen-learn-act approach 
is a possible instrument for controlling this issue. Regular "listening" is possible at an almost exclusively software-supported global, country, or location level via (semi-)structured surveys. This is already established in practice, in particular in large companies such as SAP (as mentioned in Sect. 3). Such surveys, if they are carried out regularly, can be used to implement the "listen" part of the approach presented. However, the question arises as to the evaluation of the answers obtained in the survey, especially if there are a large number of free-text answers. For the implementation of the "learn" and "act" parts, this must be done in an automated and resilient manner. We use a multistage approach for the evaluation concerning the resilience criteria for employer experience as displayed in Table 1:

- Analysis of the survey results and response texts with the help of data analysis methods, especially with regard to the topics and sentiments contained therein. Algorithms from the area of topic modeling (including latent Dirichlet allocation, Blei et al. 2003; Yin and Wang 2014; and sentiment analysis, Gilbert and Hutto 2014) are used.

- Technical and business-driven (but also partly automated) derivation of hypotheses with regard to the areas of interest (e.g., employee satisfaction, relationship between the manager and the employee, problems in everyday work of the employees).

- Verifying or falsifying the hypotheses with the help of targeted data analysis (which, e.g., are also based on the hierarchy structure).

- Combination of the data analysis results with operational data for further verification or falsification.

The last step is the most challenging but also the most important one. For example, can the challenges often mentioned by employees also be recognized in the performance of agile development teams (e.g., burndown rate)? Can correlations between financial indicators and the problems mentioned (or positive effects) be identified? If this step is carried out successfully, the organization can learn from it and act accordingly. The basic requirements for such a system to be resilient are, in addition to the criteria listed in Table 1, regular surveys and a standardized process to carry out the abovementioned four steps.

\section{Use Case 2: Manufacturing}

The manufacturing sector has undergone a tremendous digital transformation. Ubiquitous computing, connectivity combined, and integration of diverse sensors have created a next-generation industrial infrastructure (Feng and Shanthikumar 2018). These investments help manufacturing companies to further increase efficiency of production processes (Wuest et al. 2016). However, these efforts are often focused on equipment monitoring and automation solutions. Typical examples include predictive maintenance for key productive assets (McKone and Weiss 2002) or automated quality control of outputs (Flath and Stein 2017; Wuest et al. 2014). How- 
ever, oftentimes, humans still play an important role in manufacturing processes, in particular when complex or highly variant operations are necessary (assembly, customized products). Still, analytics can pave the way to resilient manufacturing processes by supporting workers to improve along the dimensions of flexibility, tolerance, and cohesion. Empowered by artificial intelligence innovations, IoT solutions stand at the forefront of digital transformation. Augmented intelligence solutions which enhance human performance in complex tasks are of particular interest. However, there are no turnkey solutions for developing and implementing such systems. One possible avenue is to complement multipurpose hardware with flexible AI solutions which are adapted to a given task.

Stein et al. (2018) describe how advanced analytics can assist human workers in a high-tech production process, namely, vacuum resin infusion. During this process, molds are placed in vacuum bags. Any leakages will impair quality. Therefore, leak detection is of highest priority. This is a manual activity (e.g., using ultrasonic microphones or thermal cameras), and therefore, the production of large components (e.g., aircraft wings) becomes extremely expensive. The research augments the standard search process by means of generic, multiuse vibration sensors collecting data during the infusion process. Subsequently, predictive models based on machine learning algorithms provide high-accuracy predictions for leak locations. Using prescriptive analytics, these predictions can be directly transformed into better search paths which can substantially reduce the work required for leak detection. This increases organizational flexibility as workers are relieved of nonproductive tasks.

Krenzer et al. (2019) illustrate the bottom-up development of a machine learning backend for an augmented intelligence system for assembly environments. A wearable sensing device is paired with a deep neural network to monitor connector systems assembly in real time. This system can monitor the correctness of assembled plugs based on structure-borne noise signals. The initial study yields promising results and establishes the feasibility of the suggested approach. Furthermore, it serves as a blueprint for similar IoT applications which facilitate improved process tolerance through high transparency.

Oberdorf et al. (2020) elaborate on the design, evaluation, and roll out of an escalation management system with integrated data-driven decision support. The decision support functionalities leverage state-of-the-art machine learning algorithms for disruption-type classification as well as prediction of the escalation handling duration. These predictions are then embedded in an integrated planning procedure leveraging diverse organizational data sources (e.g., personnel availability, production plans) to instantiate a prescriptive analytics solution. The proposed escalation management system generates significant business value by reducing escalation duration. This improvement is due to automation as well as improved decision support. In the long term, the informational and transformational business value enabled by the decision support system may even exceed the automational business value. This highlights the special importance of tight integration of Industry 4.0 applications within business processes. In particular, such a solution ensures the flexibility of troubleshooting processes and introduces a tolerant process 
control through transparency. Ultimately, analytics support improves organizational cohesion as workers complement themselves in an optimized fashion.

\section{Use Case 3: Purchasing and Logistics}

Purchasing and logistics processes are subject to fluctuations in demand. Therefore, knowledge of demand is advantageous for optimizing internal processes (warehousing, supplier management, etc.). The challenge is that demand varies over time and is different for each product. In this respect, the creation of a forecast model depends on industry- or product-specific factors. AI can help to build such forecasting models (van Dinther and Mauch 2019).

Economic advantages through forecasts in purchasing can be achieved in particular by optimizing processes. For example, storage costs can be lowered, or transports can be reduced by bundling orders or the number of short-term transports (e.g., due to subsequent orders). In addition, targeted supplier management can improve the negotiating position with suppliers and thereby achieve price advantages. An optimization of sales management is also possible.

In this case, we consider a dealer of rubber products in the B2B sector with over 20,000 customers. The ERP system records 250,000 product numbers, which can be grouped into classes so that similar products are easier to identify. The dealer purchases these products from over 2000 suppliers worldwide. The products range from simple standard articles to special products with specific properties depending on the intended use. Special products require knowledge of the properties of the materials, require intensive consultation, and are often part of a project business. For such products, forecasting is difficult and, with regard to logistics processes, also superfluous, since the lead times in projects are long enough to effectively manage internal processes. For example, customers for the construction of special machines order hoses of certain sizes and lengths for hydraulic applications, i.e., the hoses are filled with oil and must withstand certain pressures. Advice is needed here as to which types of rubber are suitable, and internal knowledge is required from which supplier they can be obtained. These special hoses are not standard goods and are therefore only ordered from the supplier after the customer has placed his order. Therefore, it is not necessary to develop a forecast for these products.

In contrast to such products, the forecast for the demand of standard goods is much more interesting, both from a business point of view and from a scientific point of view. Standard products must be in stock in sufficient quantities to be able to process customer orders reliably. However, the demand for standard goods varies greatly and ranges from continuous demand to products that are in regular but not continuous demand. This makes the forecast problem challenging.

The distribution of product order quantities is a typical long-tail distribution, i.e., few products are ordered in large quantities and continuously, while many products are ordered in varying quantities and infrequently. The latter are called lumpy time series. Especially for such lumpy time series, forecasting is difficult because both 
the time interval between orders and the order quantity vary. For the mediumterm forecast, the aim is to forecast sales at the industry and divisional level for the top 1200 customers and top 2000 articles for up to 6 months into the future. This is done using data from the past 12 months with information on the order date and the industry and division, general data such as public holidays and external data such as the ifo Business Climate Index, or industry data from the Federal Statistical Office and Google Trends. Various algorithms were implemented and compared, including naive periodic approaches, linear regression, SARIMAX, HoltWinters triple exponential smoothing, Support Vector Regression, Random Forests and Gradient Boosted Trees, multilayer perceptron artificial neural networks, or LSTM-RNN.

The procedures were compared using R2 and RMSE. It is not surprising that the naive approaches were not well suited. Similarly, bad results were obtained with SARIMAX, but good results were obtained with the other approaches. Classical forecasting methods such as Croston (1972) or Peter R. Winters (1960) perform well compared to AI-based methods. Compared to classical approaches, long shortterm memory (LSTM) neural networks show similarly good results for lumpy time series forecasting (Kiefer and van Dinther 2020). This revealed considerable savings potential. For example, the medium-term forecast of the RMSE was reduced by up to $80 \%$ compared to the previously used forecast.

High-quality forecasts are particularly valuable in the retail sector, as errors affect stock levels and delivery times. In this respect, resilient forecast models represent an important economic value. However, with regard to the three characteristics of resilient systems, there is a trade-off with forecast models. In the present case study, forecasts are mainly based on historical data, i.e., on order patterns in the past. It is assumed in the model that there are repeated time cycles. Without external sources that provide a reliable indication of a trend change or shock, it is almost impossible to anticipate abrupt events like the COVID-19 crisis. Therefore, the question on the adaptability (flexibility) of the model after an external shock is much more important, i.e., the question of how quickly the system takes up and adapts to a permanent change. The weighting of historical data in the forecast plays a role in this respect in that more recent data receive a stronger weighting than events further in the past. On the other hand, there is a risk of underweighting long-term trends or seasonal events. The aim in the forecast models is therefore to correctly determine the tolerance in the model's adjustment speed. It is necessary to take up permanent changes without at the same time distorting the forecast results by short-term deviations. With regard to the third property of resilient systems, cohesion, it should be noted on the basis of the present case study that the interaction between employees and forecast models is important. Especially in the application case with lumpy time series, this trade-off between short-term adjustment and long-term trends is difficult. In this respect, we do not yet see any possibility of fully automating orders on the basis of the forecast model results. This is where employees are called upon to check plausibility, who may come to different decisions based on context information or information that is not taken into account in the model. 


\section{Use Case 4: Software Transition}

Hyper-distribution of teams makes the management of projects even harder than it has already been. Complex transformation projects to new standard software are always a state of exception, and a pandemic potentiates this.

According to Friedman (2009), a majority of $83 \%$ of data migrations fail or significantly exceed time and budget. And this is only one part of a software transition that can go wrong. It not only hurts user adoption and the value of the new system but can cause the respective transition and transformation project to fail overall, which in turn means losing tens or hundreds of millions of euros in large transformations. It also means a traumatizing experience for project participants. Many of them typically take care of the already challenging but well-practiced job of maintaining and managing a familiar application and are in a state of exception during a transition.

Transition issues are hardly ever technical in nature. The challenges lie in translating evolving business needs into change that remains consumable for the customer organization and in the complexity of coordinating many thousands of operational activities in a compact timeframe. In addition, many organizations lack a complete understanding of circumstances to successfully undertake the complex changes required in their legacy systems to attain advantages of migration (Gholami et al. 2017; Strobl et al. 2020). Distributed teams do not make this easier. Following the megatrend of the recent past, namely, outsourcing IT services, teams are oftentimes not only distributed across different customer locations but include one or several offshore locations as well. Even before the COVID-19 pandemic, most transition projects were fragile and far from resilient with respect to timing and risk. As shown in multiple studies, delay risk prediction is one of the main concerns in the migration projects (Manekar and Gera 2017).

\subsection{Key Challenges in Transition Projects}

Inherent key challenges in such transition projects — with and without a pandemicare:

- Understanding legacy software applications and what remains relevant thereof

- Understanding how functionality and data translate from the old applications to new ones

- Reliably planning the efforts and duration of a transition endeavor

- Coordination of a multitude of tasks in configuring new software, migrating data, and taking decisions for issue resolution

- Monitoring overall progress and remaining risks

- Susceptibility to errors in the many manual activities executed by on- and offshore teams 
- Managing change and ensuring that customer organization can consume that change

Until the pandemic affected IT projects, it remained best practice to have offshore teams co-located and on-site teams at customer facilities either co-located or at least meeting physically on a regular basis. This has become impossible during full lockdowns and remains extraordinarily challenging in a hyper-distributed environment where people work primarily from home and even off-shore teams are scattered. However, all of the above challenges become even harder in a hyperdistributed world. Each of them relies on a number of decisions that are traditionally taken by humans and based on information that is compiled by humans.

\subsection{AI-Automated Platform with Consistent Digitalization}

The vision is to fundamentally change transitions for the better and address its challenges with a consistent digitalized solution. Instead of mere human experience, and individual handling of tasks and tools, a cohesive platform not only enables inherent guidance through processes, a foundation for automation in each and every task, and all the information for automatically tracking progress but is also the necessary enabler for AI to encompass the entirety of activities in transition projects. It can learn the processes, patterns, and pitfalls of projects. With enough projects captured over time, it can derive recommendations for the project participants and eventually control the automation of certain sequences of steps directly.

Building a solution begins with a consistent digital coverage of all operational processes in transition projects. This may start with the assessment of existing applications to be replaced. It continues with capturing scope and requirements, configuration of the destination applications, data profiling, data cleansing, and data migration. And it doesn't end there. The goal is to take every manual task and scattered tool, which may often be run on a local machine, and move it to a central cloud platform that guides and follows experts through a project from beginning to end. With such a foundation arise two major possibilities that were impossible before, seamless automation support of all the individual activities in a project and immediate digital insight into each and every activity, no matter if it is triggered manually or executed in a fully automated fashion. This in turn means that the latter can feed an AI with sufficiently comprehensive data, and the AI can guide more and more of the steps in the former.

Such a platform for consistent digitalization captures a unique digital fingerprint consisting of up to hundreds of thousands of data points for each project. It enables seamless collaboration and data-driven communication with real-time insight. This gives the project members a better overview and lets them see the patterns in the project across members and, hence, take smarter decisions on how to proceed. The platform also preserves the knowledge of how projects are executed in detail beyond the limited memory of the people who executed them. It retains a wealth 
of information on which projects are fast, which projects are slow, what leads to success, what leads to failure, what healthy patterns to adhere to, what unhealthy patterns to avoid, and much more. And all of this is represented in an AI-accessible format. So, the AI can draw on a wealth of information and present suggestions to the project participants that help them to take even smarter decisions faster. Having all data accessible for AI models is one of the goals we strive to tackle. As studies show in recent years, the use of AI models will only be as good as the data they are fed with. As seen in a critical case like a pandemic, not having enough meaningful data will hinder any AI technology. Collecting big enough data for transitions and transforming it into a knowledge base, which an AI model can be trained on, are the biggest challenges for every AI. Only when this is achieved, AI will reach its full potential in learning and finding insights for predictions on new projects and for smarter decisions. ${ }^{1}$

\subsection{Resilience with the Platform}

Consistent digitalization, a central home for project knowledge, and AI greatly add to the resilience of transition projects.

Flexibility is provided for a team working on such a platform both in terms of space and time. When formerly co-located teams are forced to physically isolate every individual, they can rely on a central live dashboard and status information to stay coordinated and base their decisions on facts. Team members are truly enabled to work from anywhere. Tasks and partial results can be handed over within the platform to be flexible with time zones. If certain regions are severely impaired over a longer period of time-due to issues in public infrastructure, government regulation, etc.- - all of the work can temporarily be shifted from one region to another, given that enough expert resources are available. Moreover, it is common that projects within a larger transformation initiative have dependencies on other projects for reasons like technical interfaces or expert availability. The comprehensive storage of the project work including its status also helps a team to be more flexible in adapting timelines to such external dependencies.

Tolerance is enabled, as not only all project-specific knowledge like mapping of functionality and data conversion rules is stored in a safe central place but also the most recent state of progress including every prior activity leading to the current state. Progress of the configuration of the destination applications including all fields, picklist values, workflows, and more is kept in the platform-even the latest state of source application setup: the exact state of how many records of which object have been extracted when from the source applications, converted to what extent, and imported into the destination applications at what time; how many

\footnotetext{
${ }^{1}$ More details and a commercial platform striving to fulfill this vision can be found at http://www. conemis.com.
} 
necessary activities for running all of that work have already been executed; and how many are left according to the plan. With all that knowledge in the platform, even a sudden interruption of the project by a pandemic incident can be tolerated. After such an interruption, humans participating in the project may require some time for finding orientation again and familiarizing themselves with the latest status of the project. But all of the information for that is in the system and does not depend on any distributed information on local devices. As shown in several studies, legacy data challenges and their storage during migration are key concerns that project stakeholders have in their planning and designing phase of migration (Gholami et al. 2017; Manekar and Gera 2017). In the most dire of circumstances, even a different team familiar with how the platform works in general could pick up work and continue it after some time of orientation. AI could continue with its suggestions and assistance in coordination regardless of the interruption. We nevertheless hope this will remain a merely hypothetical scenario.

Cohesion is achieved by having complete digitalization as the ultimate goal. End-to-end processes beyond functions are covered by the platform. Formerly distributed information and information solely in the heads of people is externalized and stored by the software. Interruptions of the project do not disturb cohesion in retaining the knowledge of the project. All information necessary for AI to consume remain available in a consistent form.

Cohesion is provided across projects. When leveraging a proven platform for several projects-past and present-processes and recorded experience can easily be leveraged across projects to benefit all. Experience silos, typically in the heads of a few experienced experts, are opened up for an overall increased level of quality and success in projects supported by the platform. An added benefit that is not to be neglected is that the platform automatically documents every step in every project. So even in the most exceptional and hectic of times, detailed documentation continues.

In general, existing AI approaches mainly target delay risks prediction and estimation of efforts that are unforeseen for the management team. Yet in addition, AI solutions can be the key for data protection and security of critical data that an organization has, as shown in a recent study (Diener et al. 2016). Collecting and storing big data before starting any migration is a hot topic, where the integration of AI approaches can help in handling data challenges, extractions from different resources, and designing data lakes in a migration project (Manekar and Gera 2017).

Understanding the market dynamics and evolving business needs of an organization, their translation into enabling technology, and the response to unforeseen impacts like a pandemic will require clever human minds for as far as we can see into the future. They need to take smart decisions both based on data available to them and sometimes under uncertainty. But our vision implies that all the operational tasks, including assessment, planning, configuration, data migration, and more, will sometime in the future be fully executed by an AI that builds on a vast amount of recorded knowledge to automatically orchestrate workflows with full automation in all individual tasks. This will become better with every bit of recorded project experience that can be fed into AI algorithms. Major decisions and the response 
to exceptions like a pandemic will still remain the responsibility of humans. But, although an AI-automated platform will take human direction, even in complex transformation projects, the operational execution of the transition may one day be fully autonomous.

\section{Conclusion}

The current global economic system is vulnerable to external shocks at various levels. Companies work together in value networks and are thus interdependent. Disruptions in such networks can have far-reaching consequences. Companies use IT systems to control and automate their own processes and to communicate and exchange information in value networks. As a result, the amount of digital data is constantly growing, and the demands on IT and data analysis are increasing. Technological advances in hardware and software as well as networking and sensor technology have brought major advances in the field of artificial intelligence over the last decade. Thus, AI becomes applicable in many areas and helps to advance decision-making toward smart decisions. The development of resilient systems places high demands on information systems. Thus, AI can make an important contribution in the field of smart decision-making for resilient systems.

Global-acting firms and global value networks require management attention and fast reaction on major changes such as external shocks. Therefore, more attention is to be paid to data analysis and application of AI. Such settings require firms to go the next step and embrace digitization beyond their machinery. This necessitates a paradigm shift where AI complements humans instead of replacing them (Bansal et al. 2019; Kamar et al. 2012). Such smart decision-making has been discussed in many different domains including chess-playing (Kasparov 2017), medical decision-making (Paul et al. 2018), as well as data science projects (Wang et al. 2019). Oftentimes, these human-AI teams have demonstrated higher resilience (avoiding pitfalls such as biased training data or implausible decisions) or even better performance in their respective tasks.

In our contribution, we study four use cases from very different application domains. All use cases apply AI to specific decision-making context. The interesting point to note is that the application of AI is very diverse. In the first use case, AI is applied to employee management and team development. AI analyzes text information from employee surveys in order to identify necessary actions in team development. In manufacturing, AI is applied to improve quality processes by using visual defect detection. AI is trained to identify defects (such as cracks or holes) in packaging. In sales, the focus is on improving customer order forecasts in order to optimize logistics and purchasing. The challenge here is to forecast orders for products with irregular and fluctuating demand (lumpy time series). Especially here, an optimization of resilient systems is advantageous. Inventory processes can be automated based on improved forecasting processes. AI can also be successfully used in IT projects to analyze and optimize data migration processes. Data migration 
and transformation is a critical process within IT projects due to the complexity caused by data volume and data properties. Analysis of project data can help to better manage or automate transformation processes and to identify and eliminate errors. Furthermore, AI can support the data transformation process itself.

However, the use cases also show that full automation is not yet available. However, the development advances permanently, so that an increase in the resilience of the systems becomes possible. So far technology is already advanced; it must nevertheless be stated that still further research and development remain necessary. Nevertheless, we can state that we can already use data analysis for smart decisions in resilient systems.

\section{References}

Akerkar, R. 2019. Artificial Intelligence for Business: Springer Briefs in Business. Springer.

Araujo, Theo, et al. 2020. "In AI we trust? Perceptions about automated decision-making by artificial intelligence". AI \& SOCIETY ISSN: 1435-5655. https://doi.org/10.1007/s00146-01900931-w.

Bader, Verena, and Stephan Kaiser. 2019. "Algorithmic decision-making? The user interface and its role for human involvement in decisions supported by artificial intelligence". Organization 26 (5): 655-672. ISSN: 1350-5084. https://doi.org/10.1177/1350508419855714.

Bansal, Gagan, et al. 2019. "Updates in human-AI teams: Understanding and addressing the performance/compatibility tradeoff". In Proceedings of the AAAI Conference on Artificial Intelligence, 33:2429-2437.

Blau, B., et al. 2009. "Service Value Networks". In 2009 IEEE Conference on Commerce and Enterprise Computing, 194-201.

Blei, David M, Andrew Y Ng, and Michael I Jordan. 2003. "Latent Dirichlet allocation”. Journal of machine Learning research 3 (Jan): 993-1022.

Burton, Jason W., Mari-Klara Stein, and Tina Blegind Jensen. 2020. "A systematic review of algorithm aversion in augmented decision making". Journal of Behavioral Decision Making 33 (2): 220-239. ISSN: 0894-3257. https://doi.org/10.1002/bdm.2155.

Cheema-Fox, Alexander, et al. 2020. "Corporate Resilience and Response During COVID-19". Harvard Business School Accounting and Management Unit Working Paper No. 20-108.

Croston, J. D. 1972. "Forecasting and Stock Control for Intermittent Demands". Operational Research Quarterly (1970-1977) 23 (3): 289. ISSN: 00303623. https://doi.org/10.2307/ 3007885.

Diener, Michael, Leopold Blessing, and Rappel Nina. 2016. "Tackling the Cloud Adoption Dilemma - A User Centric Concept to Control Cloud Migration Processes by Using Machine Learning Technologies". In International Conference on Availability, Reliability and Security (ARES).

Edwards, J. S., Y. Duan, and P.C. Robins. 2000. "An analysis of expert systems for business decision making at different levels and in different roles". European Journal of Information Systems 9 (1): 36-46.

Fan, Zhi-Ping, Yu-Jie Che, and Zhen-Yu Chen. 2017. "Product sales forecasting using online reviews and historical sales data: A method combining the Bass model and sentiment analysis". Journal of Business Research 74:90-100. ISSN: 0148-2963. https://doi.org/10.1016/j.jbusres. 2017.01.010. http://www.sciencedirect.com/science/article/pii/S0148296317300231.

Feng, Qi, and J George Shanthikumar. 2018. "How research in production and operations management may evolve in the era of big data". Production and Operations Management 27 (9): 1670-1684. 
Flath, Christoph M., and Nikolai Stein. 2017. "Towards a Data Science Toolbox for Industrial Analytics Applications". Computers in Industry 94:16-25.

Folke, Carl, et al. 2002. "Resilience and Sustainable Development: Building Adaptive Capacity in a World of Transformations". AMBIO: A Journal of the Human Environment 31 (5): 437-440. https://doi.org/10.1579/0044-7447-31.5.437.

Foxall, Gordon R. 2017. Advanced introduction to consumer behavior analysis. Elgar advanced introductions. Cheltenham, UK: Edward Elgar. ISBN: 1784716928.

Frank Stein and Arnold Greenland. 2014. "Producing Insights from Information through Analytics". In Business Analytics, ed. by Jay Liebowitz, 29-54. CRC Press Taylor and Francis Group.

Friedman, Ted. 2009. "Risks and Challenges in Data Migrations and Conversions". Retrieved from Gartner Research Portal.

Gholami, Mahdi Fahmideh, et al. 2017. "Challenges in migrating legacy software systems to the cloud - an empirical study". Information Systems 67:100-113. ISSN: 03064379. https://doi.org/10.1016/j.is.2017.03.008. http://www.sciencedirect.com/science/article/ pii/S0306437917301564.

Gilbert, CHE, and Erric Hutto. 2014. "Vader: A parsimonious rule-based model for sentiment analysis of social media text". In Eighth International Conference on Weblogs and Social Media (ICWSM-14). Available at (20/04/16) http://comp.social.gatech.edu/papers/icwsm14. vader.hutto.pdf, 81:82.

Golan, Maureen S., Laura H. Jernegan, and Igor Linkov. 2020. "Trends and applications of resilience analytics in supply chain modeling: systematic literature review in the context of the COVID-19 pandemic". Environment systems \& decisions: 1-22. https://doi.org/10.1007/ s10669-020-09777-w.

Gunderson, Lance H., and C. S. Holling. 2002. Panarchy: Understanding transformations in human and natural systems. Washington, DC: Island Press. ISBN: 9781559638579.

Hartmann, Nathaniel N., and Bruno Lussier. 2020. "Managing the sales force through the unexpected exogenous COVID-19 crisis". Industrial Marketing Management 88:101-111. ISSN: 0019-8501. https://doi.org/10.1016/j.indmarman.2020.05.005 http://www.sciencedirect. com/science/article/pii/S0019850120302972.

Holling, C. S. 2001. "Understanding the complexity of economic, ecological, and social systems". Ecosystems 4:390-405. https://doi.org/10.1007/s10021-00-0101-5.

Ivanov, Dmitry. 2020. "Predicting the impacts of epidemic outbreaks on global supply chains: A simulation-based analysis on the coronavirus outbreak (COVID-19/SARS-CoV-2) case". Transportation research. Part E, Logistics and transportation review 136:101922. https://doi. org/10.1016/j.tre.2020.101922.

Jackson, Scott, and Timothy L. J. Ferris. 2013. "Resilience principles for engineered systems". Systems Engineering16 (2): 152-164. doi: 10.1002/sys21228. eprint: https://onlinelibrary. wiley.com/doi/pdf/10.1002/sys. 21228

Kamar, Ece, Severin Hacker, and Eric Horvitz. 2012. "Combining human and machine intelligence in large-scale crowdsourcing." In AAMAS. 12:467-474.

Kasparov, Garry. 2017. Deep thinking: where machine intelligence ends and human creativity begins. Hachette UK.

Kiefer, Daniel, and Clemens van Dinther. 2020. "Demand Forecasting Intermittent and Lumpy Time Series: Deep Learning a magic bullet?"

Krenzer, Adrian, et al. 2019. "Augmented Intelligence for Quality Control of Manual Assembly Processes using Industrial Wearable Systems". In Proceedings of the 40th International Conference on Information Systems (ICIS).

Kusiak, A., et al. 2000. "Autonomous decision-making: a data mining approach". IEEE transactions on information technology in biomedicine: a publication of the IEEE Engineering in Medicine and Biology Society 4 (4): 274-284. ISSN: 1089-7771. https://doi.org/10.1109/4233. 897059.

Lakhmi C. Jain. 2009. "Advances in Decision Making”. In Recent Advances in Decision Making, ed. by Janusz Kacprzyk et al., 1-6. Berlin, Heidelberg: Springer Berlin Heidelberg. ISBN: 9783-642-02186-2. 
Lepenioti, Katerina, et al. 2020. "Prescriptive analytics: Literature review and research challenges". International Journal of Information Management 50:57-70. ISSN: 02684012. https://doi.org/ 10.1016/j.ijinfomgt.2019.04.003.

Loureiro, A.L.D., V. L. Miguéis, and Lucas F.M. da Silva. 2018. "Exploring the use of deep neural networks for sales forecasting in fashion retail". Decision Support Systems 114:81-93. ISSN: 01679236. https://doi.org/10.1016/j.dss.2018.08.010

Manekar, S, and Pradeepini Gera. 2017. "Opportunity and Challenges for Migrating Big Data Analytics in Cloud". IOP Conference Series: Materials Science and Engineering225 (): 012148. https://doi.org/10.1088/1757-899X/225/1/012148.

McKone, Kathleen E, and Elliott N Weiss. 2002. "Guidelines for implementing predictive maintenance". Production and Operations Management11 (2): 109-124.

Oberdorf, Felix, et al. 2020. "ADR for Big-Data IT Artifact Development: An Escalation Management Example". In Proceedings of the 41st International Conference on Information Systems (ICIS).

Paul, H Yi, Ferdinand K Hui, and Daniel SW Ting. 2018. "Artificial intelligence and radiology: collaboration is key". Journal of the American College of Radiology15 (5): 781-783.

Peter R. Winters. 1960. "Forecasting Sales by Exponentially Weighted Moving Averages". Management Science6 (3): 324-342.

Phillips-Wren, G., and L. Jain. 2006. "Knowledge-based intelligent Information and Engineering Systems". Chap. Artificial Intelligence for Decision Making, ed. by Bogdan Gabrys, Robert J. Howlett, and Lakhmi Jain, 531-536. Springer.

Phillips-Wren, G. (2012).Phillips-Wren, Gloria. 2012. "AI tools in Decision Making Support Systems: a review". International Journal on Artificial Intelligence Tools21 (02): 1240005. ISSN: 0218-2130. https://doi.org/10.1142/S0218213012400052.

Pierre Haren and David Simchi-Levi. 2020. "How Coronavirus Could Impact the Global Supply Chain by Mid-March". Harvard Business Review2020 (03).

Ricardo, David. 1817. The Principles of Political Economy and Taxation. Reprint from 1926. London and Toronto: J.M. Dent/Sons.

Sharma, Amalesh, Anirban Adhikary, and Sourav Bikash Borah. 2020. "Covid-19/s impact on supply chain decisions: Strategic insights from NASDAQ 100 firms using Twitter data". Journal of Business Research117:443-449. ISSN: 0148-2963. https://doi.org/10.1016/j.jbusres. 2020.05.035. http://www.sciencedirect.com/science/article/pii/S0148296320303210.

Shrestha, Yash Raj, Shiko M. Ben-Menahem, and Georg von Krogh. 2019. "Organizational Decision-Making Structures in the Age of Artificial Intelligence". California Management Review61 (4): 66-83. ISSN: 0008-1256. https://doi.org/10.1177/0008125619862257.

Stein, Nikolai, Jan Meller, and Christoph M Flath. 2018. "Big data on the shop-floor: sensor-based decision-support for manual processes". Journal of Business Economics88 (5): 593-616.

Strobl, Stefan, Mario Bernhart, and Thomas Grechenig. 2020. "Towards a Topology for Legacy System Migration". In Proceedings of the IEEE/ACM 42nd International Conference on Software Engineering Workshops, 586-594. IC-SEW'20. Seoul, Republic of Korea: Association for Computing Machinery. ISBN: 9781450379632. https://doi.org/10.1145/3387940.3391476.

Stubbs, Evan. 2014. "Business Analytics: An Introduction". Chap. The Value of Business Analytics, ed. by Jay Liebowitz, 1-28. CRC Press, Taylor / Francis Group.

Wang, Dakuo, et al. 2019. "Human-AI Collaboration in Data Science: Exploring Data Scientists' Perceptions of Automated AI". Proceedings of the ACM on Human-Computer Interaction3 (CSCW): 1-24.

Wuest, Thorsten, Christopher Irgens, and Klaus-Dieter Thoben. 2014. "An approach to monitoring quality in manufacturing using supervised machine learning on product state data". Journal of Intelligent Manufacturing25 (5): 1167-1180.

Wuest, Thorsten, et al. 2016. "Machine learning in manufacturing: advantages, challenges, and applications". Production \& Manufacturing Research4 (1): 23-45.

Yin, Jianhua, and Jianyong Wang. 2014. "A Dirichlet multinomial mixture model-based approach for short text clustering". In Proceedings of the 20th ACM SIGKDD international conference on Knowledge discovery and data mining, 233-242. 
van Dinther, Clemens. 2007. Adaptive Bidding in Single-Sided Auctions Under Uncertainty: An Agent-based Approach in Market Engineering. Whitestein Series in Software Agent Technologies and Autonomic Computing. Basel: Birkhaäuser Verlag. ISBN: 978-3764380946.

- 2008. "Agent-based Simulation for Research in Economics". In Handbook on Information Technology in Finance, ed. by Detlef Seese, Christof Weinhardt, and Frank Schlottmann, 421442. Berlin, Heidelberg: Springer Berlin Heidelberg. ISBN: 978-3-540-49487-4. https://doi. org/10.1007/978-3-540-49487-4_18.

van Dinther, Clemens, and Svenja Mauch. 2019. "Chancen der künstlichen Intelligenz zur Prognose im Mittelstand”. Decision Growth, no. 3: 21-27. https://decision-growth.de/Magazin// catalogs/Growth_Magazin_III/growth-Magazin-Ausgabe-3/pdf/complete.pdf.

Open Access This chapter is licensed under the terms of the Creative Commons Attribution 4.0 International License (http://creativecommons.org/licenses/by/4.0/), which permits use, sharing, adaptation, distribution and reproduction in any medium or format, as long as you give appropriate credit to the original author(s) and the source, provide a link to the Creative Commons license and indicate if changes were made.

The images or other third party material in this chapter are included in the chapter's Creative Commons license, unless indicated otherwise in a credit line to the material. If material is not included in the chapter's Creative Commons license and your intended use is not permitted by statutory regulation or exceeds the permitted use, you will need to obtain permission directly from the copyright holder. 